\title{
Théologiques
}

Théologiques

\section{Le croyant singulier et invisible}

\section{Vincent Delecroix}

Volume 16, numéro 2, 2008

L'individualisation de la relation religieuse

URI : https://id.erudit.org/iderudit/001716ar

DOI : https://doi.org/10.7202/001716ar

Aller au sommaire du numéro

Éditeur(s)

Faculté de théologie et de sciences des religions, Université de Montréal

ISSN

1188-7109 (imprimé)

1492-1413 (numérique)

Découvrir la revue

Citer cet article

Delecroix, V. (2008). Le croyant singulier et invisible. Théologiques, 16(2), 87-104. https://doi.org/10.7202/001716ar

\section{Résumé de l'article}

À trop faire porter l'accent sur la relation individuelle et subjective à son objet, la pensée philosophique qui s'attache au religieux peut en venir à des cas limites où la nature sociale de la religion lui devient, d'un point de vue épistémologique, difficilement visible et pensable. On se proposera ici d'examiner les formes de cette articulation problématique entre expérience singulière et communautés objectives dans le cadre d'une comparaison entre deux de ces cas limites : Schleiermacher et Kierkegaard.
Ce document est protégé par la loi sur le droit d'auteur. L'utilisation des services d’Érudit (y compris la reproduction) est assujettie à sa politique d'utilisation que vous pouvez consulter en ligne.

https://apropos.erudit.org/fr/usagers/politique-dutilisation/ 


\title{
Le croyant singulier et invisible
}

\author{
Vincent DELECROIX \\ Sciences religieuses \\ École Pratique des Hautes Études
}

En choisissant de mettre en regard la pensée de F. Schleiermacher (1768$1834)$ et celle de S. Kierkegaard (1811-1855), nous voudrions comparer et confronter deux approches philosophiques du religieux qui, plaçant un net accent sur l'individualisation de la relation religieuse, se voient contraintes de repenser la nature d'une communalisation religieuse, elle-même objet, comme institution, d'une critique radicale. Protestants, fortement marqués par le piétisme, liés de manière critique au romantisme allemand, en rupture également avec "l'Église établie », ils fournissent par leur pensée l'exemple significatif d'une intériorisation et d'une individualisation de la religion, caractéristique de ce moment à la fois religieux et philosophique. Cette première communauté de pensée laisse cependant apparaître une divergence de plus en plus nette quant à la manière de concevoir l'articulation entre la relation individualisée à l'absolu et l'établissement d'une communauté de croyance: la comparaison doit ainsi servir à mettre en relief les catégories philosophiques par lesquelles peut être pensée cette articulation, catégories qui en outre peuvent se révéler opératoires pour une analyse de la modernité religieuse, si l'un des traits de cette modernité est bien l'individualisation de la relation et la reconstitution problématique de la communauté religieuse à partir de cette individualisation.

On commencera ainsi par montrer en quoi, et comment, la singularisation de la relation à Dieu (ou à l'Infini, dans le cas de Schleiermacher) constitue pour ces deux penseurs le contenu fondamental du phénomène religieux. Il s'agira particulièrement de décrire comment, dans ce cadre

* Vincent Delecroix est maître de conférences en philosophie à l'École Pratique des Hautes Études (Section des Sciences religieuses). Ses recherches actuelles portent sur la question de la vérité en philosophie de la religion, à partir de la philosophie analytique et de la tradition herméneutique (théorie de la vérité et discours religieux). Il a publié, en 2006, Singulière philosophie. Essai sur Kierkegaard, Paris, Le Félin. 
conceptuel, se constitue la notion d'individu religieux. On tâchera ensuite, toujours par le biais de cette comparaison, de décrire et d'analyser la nature de la relation elle-même, c'est-à-dire le type d'opération qui la rend effective. Cette opération - expressivité (ou effusion) pour l'un, imitation pour l'autre - fournit la face visible d'une religiosité pourtant pensée sur le mode de l'intériorisation et c'est sur cette dialectique entre l'invisibilité de la religiosité "profonde » et sa face nécessairement visible qu'il faudra insister. Or, c'est cette face visible (ou problématiquement visible) qui doit servir de socle à la constitution de la communauté. La communauté se constituant à partir d'une communication de la relation individuelle (ou du «sentiment» religieux), on tâchera donc en dernier lieu de comprendre les lois ou les traits caractéristiques de cette communication. Sur ce dernier plan, Schleiermacher et Kierkegaard se trouvent à des pôles presque radicalement opposés, la religiosité chez Schleiermacher se diffusant par propagation du sentiment, tandis que, isolant toujours plus l'individu, selon Kierkegaard, elle n'autorise qu'une communication et une compréhension indirectes. On tâchera ainsi, pour finir, d'esquisser les deux paradigmes respectifs de cette communication religieuse qu'il faut effectivement comprendre à partir du cadre discursif. Deux paradigmes qui sont aussi deux arts d'écrire, par lesquels pourrait se créer cette communauté religieuse authentique.

\section{De la relation singulière}

Schleiermacher et Kierkegaard peuvent représenter des cas limites dans la philosophie de la religion. Dans les deux cas, en effet, on voit surgir comme élément problématique fondamental les conséquences périlleuses induites par une focalisation du religieux sur l'individu, et plus précisément sur la relation du sujet à son objet, le Dieu personnel pour Kierkegaard ou l'Univers, au contraire dépersonnalisé, pour Schleiermacher ${ }^{1}$. Certes, pour aucun des deux, la face visible et institutionnelle de la religion, malgré la critique dont elle est l'objet (à des titres différents), ne peut être écartée ou tenue pour négligeable: le caractère communautaire de la religion nécessite d'être compris, mais il ne peut l'être qu'à partir d'une définition du religieux qui semble paradoxalement en interdire la pleine légitimité.

1. Voir Schleiermacher $(2004,73)$ : «Dieu n'est pas tout dans la religion, mais l'un de ses éléments, et l'Univers, est davantage que lui. » 
Or, les réponses peuvent être opposées. Si elles partent toutes les deux d'une critique de la religion instituée, pour l'un, Schleiermacher, la religion a une dimension fondamentalement sociale (et historique) qu'il s'agit dès lors de "recomprendre " adéquatement (précisément pour éviter les erreurs de la religion instituée actuelle ${ }^{2}$ ), tandis que pour l'autre, Kierkegaard, le lien à la communauté ecclésiale reste fondamentalement conflictuel, même si l'institution sociale de la religion relève, semble-t-il, d'une loi historique irréfragable. Il reste toutefois que, pour l'un comme pour l'autre, le problème de la visibilité de cette relation devient central, ce qui les contraint à penser de façon originale la religion et non plus seulement l'expérience religieuse: quels signes manifestent la relation religieuse authentique? De quelle nature sont ces signes? Le problème général pourrait ainsi se concentrer dans la question suivante: Comment peut-on passer d'une expérience religieuse conçue comme absolument singulière et individuelle à l'institution d'une religion?

Mais quelle est donc cette relation singulière? Les termes sont parlants: sentiment de dépendance absolue (Schleiermacher), être devant Dieu (Kierkegaard). Dans le premier cas, celui de Schleiermacher, le sentiment individuel — mais universellement partagé, puisqu'il appartient à la nature humaine en tant que "province autonome de l'esprit» — est, si l'on peut dire, l'alpha et l'oméga du religieux. À la fois racine et expression achevée, à la fois simple puissance d'être affectée, actualisée selon des modes à chaque fois individuels - et, évidemment, avec des degrés d'intensité variable -, il est aussi le terminus ad quem de l'expérience authentiquement religieuse, celle des «virtuoses». De même, infra-linguistique, il peut parvenir, dans des modes individualisés adéquats, à des expressions complexes, lesquelles se refroidiront dans le dogme et le Texte, qui sont comme des " mausolées $^{3} »$. Il y rencontre d'ailleurs là une limite fondamentale, le médium du livre et plus généralement encore du signe linguistique se révélant partiellement inadéquat ${ }^{4}$.

Or, cette singularité du sentiment, qui concentre l'essence de la religion, ouvre à un dépassement de la personnalité dont il est issu. Non pas que le sujet individuel fusionnerait avec l'Infini, puisqu'il est déterminé par une passivité ontologique irréductible à cet égard (et d'autant plus que ce

2. Tout le quatrième discours de De la religion y est consacré.

3. Schleiermacher 2004, 67.

4. Voir Schleiermacher $(2004,101-102)$ : «Une part trop importante de l'impression originelle se perd dans l'emploi de ce médium où se trouve englouti tout ce qui n'est pas compatible avec ce que les signes ont d'uniforme. » 
qu'il « intuitionne », dans l'intuition de l'Infini, c'est d'abord sa nature de créature), mais il se comprend ou se représente lui-même comme "compendium de l'humanité » (Schleiermacher 2004, 54). C'est qu'il parvient effectivement à une vision de l'activité infinie qui oblige à dépasser les unités discrètes et individuelles: "contempler et considérer ces objets dans le cadre du Tout, et non comme quelque chose de séparé et d'auto-suffisant » (63). Le paradoxe veut donc que l'accentuation de l'individuum (un «individuum exceptionnel » qualifie le virtuose) ait pour effet, dans le sentiment religieux, de le dés-absolutiser. Pourtant, la limite discrète qui sépare les individus dans l'expérience religieuse n'est pas transgressée: le sentiment reste purement individuel, il s'exprime toujours selon un mode particulier irréductible aux autres. En outre, jamais un tel dépassement de l'individu dans l'individuel ne se fait sous la forme d'une union fusionnelle. Schleiermacher trouve dans l'expérience religieuse un principe que son herméneutique générale placera à son commencement: la relation de la partie au Tout et du Tout à la partie, premier cercle herméneutique particulièrement visible dans la relation religieuse. L'individu ne se «fond" pas dans le Tout: ce qu'il comprend, c'est précisément la relation elle-même qui, circulaire, lie la partie au Tout.

D'autres conséquences sont plus spectaculaires encore. La particularité même de l'expérience, son ancrage dans un situs, oblige à considérer comme nécessaire non seulement la variété virtuellement infinie de ces expériences, mais aussi leur égale prétention à l'authenticité. La pluralité des religions est donc l'expression de la pluralité nécessaire des sentiments; elle n'est pas une fragmentation désastreuse et historiquement contingente d'une religion naturelle universelle, laquelle n'est qu'une chimère métaphysique de philosophe (139-140 et 160; voir Simon 1974, 261-265), elle est l'expression même de l'essence de la religion. $L a$ religion ne peut pas être, justement, une. Ce qui a pour conséquence également que la constitution d'un dieu personnel comme objet du sentiment religieux, «Dieu ", n'est qu'une expression possible du sentiment. Autrement dit, la relation par essence individuelle n'a pas pour corrélat la personnalisation ou l'individuation de son objet: "la Divinité ne peut rien être d'autre qu'une forme particulière d'intuition religieuse dont les autres sont indépendantes, comme elles le sont toutes les unes des autres » (69). Et Schleiermacher de déclarer : «Et pourquoi donc devrais-je accorder plus d'importance à un mode d'intuition religieuse qu'à d'autres ?» (68). Il n'y a pas de liaison nécessaire entre une expérience individuelle et un Dieu personnel. Tout dépend, dit Schleiermacher, de 
l'orientation de l'imagination (71). L'objet de l'intuition, ce qu'il nomme aussi «l'Univers", est "plus » que Dieu. Certes, il est possible d'évaluer ces intuitions, mais une telle évaluation pourrait aussi bien nous amener à considérer «qu'une religion sans Dieu peut être meilleure qu'une religion avec Dieu» (70).

Par voie de conséquence, la relation communautaire originale qui caractérise la religion de Schleiermacher doit comprendre à la fois l'irréductibilité des expériences, leur différence qualitative, la communauté intermédiaire qui rapproche dans une religion instituée certaines formes de cette intuition, et la communauté de la «vraie» religion, laquelle est une dimension essentielle du sentiment religieux dans la mesure où celui-ci veut par nature se communiquer (100 sq.). Cette difficile dialectique se joue dans le paradoxe qui veut que la religion soit d'essence sociale et qu'à la fois elle trouve son origine dans cette relation irréductiblement singulière: ce qu'il faut donc penser, c'est l'idée d'un sentiment partagé. Ce sont les règles mêmes de ce "partage » qui établissent la communauté, avec l'idée qu'il y aurait, d'une part, ce qu'on pourrait appeler une règle « inférieure » de partage, celle qui caractérise les religions instituées, établies à partir d'un sentiment refroidi et dans une communauté de dogme et, d'autre part, une règle "supérieure » qui établit une communication et une communauté sur le plan même des expériences singulières ${ }^{5}$.

Cette dialectique n'est assurément pas celle de Kierkegaard, pour lequel, cependant, la relation singulière constitue le fondement absolu de la religiosité. Celle-ci revêt la double dimension d'une exigence et d'une dynamique d'individualisation. Car on aurait évidemment tort de ramener la conception kierkegaardienne à une simple subjectivation de la relation à Dieu, nécessitée par la position préalable d'un sujet pré-constitué : la relation est moins singulière que singularisante, ce qu'exprime parfaitement l'idée kierkegaardienne du "devenir chrétien ». Le sujet premier est seulement un sujet brut, simple être là posé dans sa contingence et sa finitude; autre chose est la subjectivité qui se construit (qui devient) dans la relation elle-même, qui devient, pour reprendre les termes de Kierkegaard, un Singulier (en Enkelte) devant Dieu (voir Clair 1993, 173 sq.). Le concept de Singulier dénote non pas le sujet individuel en lui-même, mais la subjectivité qui se construit (s'approfondit) dans la relation. Le «Devant

5. Sous ce vocable de règle, il faudrait comprendre à la fois les règles de la communication et les lois d'organisation de la communauté : la métaphore politique (mais c'est plus qu'une métaphore) est celle qui est retenue par Schleiermacher $(2004,105)$. 
Dieu ", qui est l'essence de la religiosité, n'est pas un mode d'être parmi d'autres du sujet: c'est son effectuation comme subjectivité singulière, le terme de son devenir (l'accomplissement du devenir-soi), laquelle ne peut donc se comprendre que comme pôle de la relation produit par la relation elle-même. Il y a donc à la fois l'exigence d'établir une relation singulière, non médiatisée, à l'Autre absolu, exigence qui est celle de la religiosité authentique, et l'exigence d'être une subjectivité concrète dont la relation religieuse est le moteur, une exigence du devenir-soi. Il faut ainsi différencier le sujet-source de la relation de la subjectivité-effet (le Singulier) qui est la pointe de l'existence - l'intériorité. Et ceci ne peut se comprendre que si l'on voit que la relation est une relation de singulier au Singulier (absolu): le religieux B, pour reprendre la terminologie kierkegaardienne du Postscriptum (spécifiquement chrétien), accentue radicalement cette relation singulière au Singulier, précisément parce que son objet est l'événement même de l'Incarnation, le sujet singulier qu'est le Christ, non pas l'HommeDieu en général, mais cet homme, singulier, qui est Dieu. Autrement dit, l'essence du religieux vrai n'est effective que lorsqu'elle s'établit sur une structure à deux polarités singulières.

Or, précisément, le marqueur de cette religiosité qui accomplit la subjectivité est l'isolement: bien au contraire de susciter un lien communautaire, la relation religieuse retranche l'individu et interdit tout "partage ", toute mise en commun d'une expérience qui ne se développe que dans le secret de l'intériorité (ou plutôt dans l'intériorité comme secret). Il ne manque certes pas de marqueurs «subjectifs» (intensité, passion, souffrance, etc.), mais la marque objective se signale d'abord dans l'éclatement de la communauté croyante au profit d'une foi isolante.

Significativement, le mode effectif de cette relation est la situation de " contemporanéité ", laquelle pulvérise la médiation de l'Église instituée et de l'histoire elle-même, et exige le retranchement par rapport à la croyance «en masse ». La foi n'est pas une croyance partagée: je suis rigoureusement seul dans la décision de croire. Ce retranchement ne se fait cependant pas au profit d'une union directe à l'Absolu singulier, bien au contraire: la situation de contemporanéité est une relation à un Autre dont me sépare un abîme infranchissable. L'idée d'une non-médiatisation de la relation (par la tradition, par l'institution, par l'histoire, par le savoir) ne produit donc en rien une communauté directe avec l'objet. C'est même le contraire, puisqu'elle fait ressortir l'incommensurabilité essentielle qu'avait tendance à relativiser la médiation du lien communautaire et de ses productions (ins- 
titution, dogme, etc.) : c'est la raison pour laquelle, dans cette relation singulière, l'objet de la relation apparaît sous la forme du paradoxe (signe de contradiction) et la relation est elle-même pathétiquement qualifiée. Son intensité, qui est le critère de son authenticité (critère donc subjectif), tient précisément à l'épreuve de cette distance: le sujet, parce qu'il est au plus près, s'éprouve comme étant en vérité au plus loin.

Mais il faut se méfier une seconde fois de la notion d'immédiateté: le rapport Dieu, dans l'approfondissement de l'intériorité, n'est évidemment pas une vision directe de Dieu (qui est « incognito », car incarné), ce qui serait le propre de l'idolâtrie et du paganisme (Kierkegaard 1977, 224-225) - Dieu étant reconnaissable objectivement et ne demandant donc pas le saut de la foi (Kierkegaard 2006, 171 sq.). S'il y a bien une "médiation » à ce rapport non médiatisé, si l'on peut dire, c'est celle de la Parole. Et la relation singulière, dans l'exacerbation de la sola scriptura, la situation de contemporanéité (2006, 104 sq.), est un principe herméneutique qui implique des règles strictes de lecture et de rapport existentiel à la Parole. La situation de contemporanéité est ainsi un réquisit herméneutique, relatif à la fois à une herméneutique du texte et à une herméneutique universelle (rapport existentiel de compréhension; voir Delecroix 2002, 243-256).

Dès lors, il semble bien que le problème auquel est confrontée la conception kierkegaardienne est celui de la visibilité même de cette relation: indubitable dans le secret de l'intériorité par les marqueurs pathiques qui la signalent, elle ne peut se laisser voir objectivement, puisqu'elle relève de l'ordre du subjectif. Le Chevalier de la foi est invisible (Kierkegaard 1972, 131). Cela serait de peu d'importance d'ailleurs, si Kierkegaard ne se préoccupait pas de déceler dans l'espace objectif des marques de cette religiosité authentique. Or, il s'en préoccupe effectivement, au moins dans le but de débarrasser la "chrétienté établie » du paganisme rampant qui, selon lui, l'affecte, car le secret de l'intériorité peut être finalement l'excuse commode que se donne l'absence même de religiosité.

Mais ce problème, qui va tant préoccuper Kierkegaard dans sa critique de plus en plus acerbe de «l'Église », dépasse la seule dialectique de l'intérieur et de l'extérieur. L'interrogation sur la visibilité de la relation intensive individuelle n'est pas en effet accidentelle : là se joue la possibilité d'une articulation entre cette relation singulière et l'institution d'une religion dont la visibilité est un trait définitoire. Pour le dire autrement, une communauté de croyance ne peut s'établir que dans et par la manifestation. À cet égard, la difficulté kierkegaardienne est très significative: on ne peut se contenter 
d'une invisibilité radicale, non pas parce qu'elle aurait besoin de se voir confirmée par des signes objectifs, non pas seulement pour éviter que le refuge dans l'intériorité soit un prétexte pour ne rien sacrifier à l'exigence de la religiosité authentique, mais parce qu'il faut bien, malgré tout, que cette religiosité s'exprime dans l'effectivité de l'existence. Une religion purement privée pourrait être justement une tentation. Et si la relation à Dieu, pour le dire brièvement, ne regarde que l'individu, elle n'en exige pas moins, d'une manière ou d'une autre, d'être pensée aussi dans son rapport aux autres relations individuelles. Certes, Kierkegaard insiste particulièrement sur la dénaturation qu'implique le caractère social de la religion, mais il ne peut faire autrement que de s'interroger sur le type de lien qui peut s'établir entre les rapports individuels à Dieu. Il doit penser non pas seulement la religiosité ou le religieux (en tant que mode spécifique de la relation à la Vérité), mais légalement la religion — non pas seulement le lien vertical, mais également le lien horizontal.

Il ne s'agit pas alors seulement de savoir que la relation verticale se fait singulièrement, il faut qualifier encore ce mode de relation singulière, c'est-à-dire établir quelle opération s'effectue.

\section{Expression, imitation}

Là se joue de nouveau une différence fondamentale. Chez l'un (Schleiermacher), la relation est intuition. Et comme telle, elle relève d'une faculté réceptive (comme chez Kant, ce qui peut précisément le différencier non seulement des schwärmerer mais aussi de la postérité postfichtéenne). Mais, contrairement à Kant, celle-ci ouvre à quelque chose comme une saisie effective, bien que nécessairement partielle, de l'objet absolu, à savoir l'Infini. Cette caractéristique ne nous intéresse ici qu'en raison des effets «horizontaux» que peut causer ce type de relation. La visibilité ou la communication d'une telle relation ne peut se faire en effet que sur le mode de l'expression directe de cette intuition, d'une expression dont la nécessité tient à la nature même de l'intuition ${ }^{6}$. La relation à l'Infini ne demeure justement pas invisible, elle profuse au contraire, sur le mode de l'expression. Ce faisant, elle rencontre les autres intuitions sur deux modes qui en sont les conséquences naturelles: soit la contamination (l'ex-

6. Voir Schleiermacher $(2004,100)$ : «L'homme doit extérioriser et communiquer ce qui est en lui, et plus une chose le motive fortement et touche profondément à son être, plus vigoureux est aussi son désir d'en voir la force se manifester en d'autres que lui.» 
pression diffuse le sentiment religieux), soit l'écho (le sentiment religieux trouve dans d'autres expressions des analogies avec la sienne). Ainsi la constitution de la communauté se fait-elle - c'est une conséquence logique - par voie d'affection : cette affection active, confirme ou même réactive la faculté réceptive. La communauté devient ainsi une sorte de totalité harmonique, voire musicale, où les expressions n'ont pas entre elles un lien de causalité directe, mais s'établissent dans un concert expressif assonant (puisqu'elles expriment toutes, mais de leur point de vue, l'Univers ou l'Infini).

Le schéma kierkegaardien est tout autre. Au contraire de la réceptivité propre au sentiment schleiermacherien, il se caractérise par une double activité. La première activité est le rapport à la Parole, dans la situation de contemporanéité. Le modèle est fourni par l'Épître de Jacques (l'Écriture comme miroir), dont Kierkegaard fait un commentaire poussé, et qui lie définitivement la relation à l'opération herméneutique fondamentale du "se comprendre soi-même ": première " activité » dans laquelle la relation religieuse constitue en quelque sorte le terminus sans cesse recommencé. Le principe de la réflexion (au sens optique) est fondamental — la Parole est un miroir - mais il se double d'un autre principe herméneutique qu'on pourrait, dans le vocabulaire de l'herméneutique contemporaine, nommer " application », et que Kierkegaard détermine spécifiquement comme imitation. C'est là, peut-être, que se trouve la clef. Un tel principe herméneutique, l'imitation, relève effectivement du pragmatique : la compréhension est application. Mais l'important est surtout la modification fondamentale qu'elle induit dans le rapport à l'objet. Elle est non représentationnelle elle est d'autant moins représentationnelle que l'objet en question est incognito, c'est-à-dire qu'il ne se manifeste pas comme ce qu'il est (l'Absolu), mais comme le contraire de ce qu'il est. L'image est empêchée (fin de l'idolâtrie), l'objet ne se voit pas (Kierkegaard 1977, 235-226). Le rapport d'imitation a affaire non pas à une image (Billede) mais à un modèle (Forbillede): celui-ci n'est pas de l'ordre de la représentation, pas même de l'exemple, mais de l'ordre du signe (de contradiction) à interpréter activement (dans l'existence).

Ce passage à l'idée de modèle s'effectue lorsque l'on comprend que ce à quoi on se rapporte n'est pas un corps de propositions à savoir, mais un sujet ou une personne: c'est l'ensemble du schème épistémique qui est alors brisé. À la question « qui est mon prochain ? ", le Christ ne répond pas par une définition, mais par une mise en scène (parabole) de personnages et conclut "Va et fais de même » : à la question épistémique (qui ? qu'est-ce que ?) est 
donnée une réponse pragmatique. Mais dès lors que le schème classique est brisé, c'est la subjectivité singulière qui est sollicitée (alors qu'elle s'efface dans le savoir, fût-il d'un ordre supérieur): l'imitation est alors le contraire d'une identification, car non seulement elle laisse subsister la différence incommensurable entre le modèle et l'imitateur, mais elle favorise la singularisation. Le modèle tire donc sa nature de modèle de son opposition à l'image. Ce faisant, il suscite non seulement un brouillage définitif de l'opération de voir (dont relève évidemment l'intuition et toute forme de savoir), mais aussi un rapport purement actif de singularisation dans l'imitation: le Forbillede est le moteur de la «Bildung».

La communauté des croyants, si communauté il y a, ne peut donc consister qu'en une communauté d'imitateurs singuliers (et qui se singularisent dans l'imitation). L'Exercice en christianisme insiste ainsi significativement sur la différence entre les admirateurs (ceux qui ont justement affaire à l'image, à un Dieu visible comme Dieu) et les imitateurs qui ont affaire non seulement à un modèle, mais à un modèle paradoxal qui conteste l'image (le modèle n'est pas Dieu, mais Dieu incognito; Kierkegaard 2006, 283 sq.).

\section{De la communauté}

Comment dès lors envisager une communauté ? Quelle place pour l'institution dans ces deux conceptions? L'évolution de la pensée schleiermacherienne de la religion tend à relativiser le contraste originel entre la religiosité et l'Église instituée qu'établissait, en partie pour des raisons stratégiques et rhétoriques, les discours de De la religion ${ }^{7}$. Au contraire, dans le cas de Kierkegaard, l'accent mis sur la relation imitative ne peut que susciter un rapport critique à la religion instituée, lequel s'exacerbe radicalement dans la polémique ultra-violente qu'il mène contre l'Église danoise de son temps. L'Église, non seulement n'est pas le lieu du religieux, mais elle en constitue même l'obstacle et la perversion, le produit d'une histoire catastrophique de la vérité qui voit celle-ci dégénérer en doctrine et la relation subjective intense se dissoudre dans des médiations objectives où le sujet renonce à se faire subjectivité. L'adoucissement du christianisme, les prêches lénifiants des pasteurs, la socialisation même de la relation religieuse ne sont que les effets d'une dégénérescence essentielle qui voit notamment la situation de

7. Voir Simon $(1974,235)$ : «Dans la Kurze Darstellung et dans la Glaubenslebre la notion d'Église jouera plus tard un rôle si central qu'on ne peut imaginer qu'elle ait jamais été mise en question par l'auteur de ces ouvrages théologiques. » 
contemporanéité étouffée sous le poids de l'histoire mondiale et objective (et de l'histoire de l'Église en particulier). La religion, c'est le mal, à la fois anthropologique et historique, qui éteint le religieux. Elle signifie la dilution du rapport de Singulier à Singulier absolu.

La critique de Schleiermacher à l'égard des Églises est certes elle aussi nécessaire, du moins dans le point de vue adopté par les discours de De la religion. L'institution des Églises paraît au mieux inutile à l'esprit religieux: «plus les hommes croissent en religion, plus l'Église leur devient indifférente, et les plus pieux se séparent d'elle avec froideur " (Schleiermacher 2004, 112). On trouve là, entre autres choses, une loi de proportionnalité inverse entre l'intensité qualitative du sentiment religieux et le partage quantitatif de la croyance, loi qu'on retrouve également chez Kierkegaard. Le constat n'est alors pas moins radical: «on ne fait partie de cette association [l'Église] que parce qu'on n'a pas de religion, et l'on y reste aussi longtemps qu'on n'en a point » (2004, 112 ; nous soulignons). L'Église est, au mieux, une communauté de non-croyants qui cherchent à croire et qui se regroupent (et se réchauffent) autour de celui qui constitue un foyer authentique, avéré, du sentiment religieux, à savoir le virtuose. Mais ce qui sous-tend un tel constat est paradoxalement, chez Schleiermacher, la conception du caractère nécessairement social de la religion. Comment lever cette contradiction apparente? C'est qu'il y a dans l'essence même du sentiment religieux une tendance à la communication. Simple aspiration à une communauté idéale? Schleiermacher précise au contraire que cette communauté, la communauté de virtuoses, est réelle ${ }^{8}$. Et nous entrons là dans une nouvelle différenciation intéressante des deux modèles que nous examinons ici.

Chez l'un comme chez l'autre, la relation individuelle, le croyant singulier, est le centre focal de la religion. Chez l'un comme chez l'autre, cette « révolution copernicienne » implique nécessairement une critique de la religion instituée comme "fausse communauté » ou comme communauté non religieuse ${ }^{9}$. Mais l'idée du caractère par essence social de la religion qu'on

8. Voir Schleiermacher $(2004,109)$ : «Tous les hommes vraiment religieux, pour autant qu'il y en eut jamais, ont colporté avec eux non seulement la foi, mais aussi le vif sentiment d'une réunion de ce type, et ils y ont réellement vécu.»

9. Voir Kierkegaard $(2006,268)$ : «Un semblable concept comme celui de "communauté" [...] est proprement une anticipation impatiente de l'éternité. [...] Chrétiennement, on lutte constamment au niveau du singulier, car c'est justement l'esprit, que chacun soit un singulier devant Dieu, que la "communauté" ait une détermination inférieure au "singulier" que chacun peut et doit être. " 
trouve chez Schleiermacher implique des règles originales de la constitution sociale et objective de la religion. Cette idée repose sur la nature nécessairement communicative et expressive du sentiment religieux: «l'homme doit extérioriser et exprimer ce qu'il y a en lui, et plus une chose le motive fortement et touche profondément à son être, plus vigoureux est aussi son désir d'en voir la force se manifester en d'autres que lui » (Schleiermacher 2004, 100). Cela signifie deux choses. D'une part, l'esprit religieux cherche chez les autres des échos et des confirmations de son propre sentiment; il est ainsi attiré, comme par attraction magnétique, vers les autres expressions individuelles du sentiment dans lesquelles il peut reconnaître l'écho de sa propre expérience. D'autre part, il y a en lui une tendance à la communication, c'est-à-dire à la propagation du sentiment religieux; c'est la raison pour laquelle tout individu réellement religieux est "prêtre » — et, inversement, le prêtre ne peut être qu'un virtuose du sentiment religieux. Ceci établit les principes mêmes de l'institution de la religion authentique: à un niveau intermédiaire, c'est-à-dire dans le cadre des religions instituées, il faut que les prêtres soient des virtuoses, autour desquels s'agglomèrent les non-croyants en quête d'un échauffement de leur propre sentiment religieux (l'effusion du virtuose produit en eux un écho); à un niveau supérieur, c'est la communauté même de virtuoses, véritable "république » où chacun est tour à tour membre du peuple et chef (105). Cette communauté est réelle et objective, alors même qu'elle ne passe par aucune institution. Elle est certes d'une certaine manière "à distance ", mais pas à la manière kierkegaardienne, car elle est fondée sur une reconnaissance et une communication directe. «Il veut des témoins, il veut que d'autres y participent » (101). Cette double tendance est à l'origine de la constitution non pas idéale mais bien réelle d'une authentique communauté de croyants, celle des virtuoses.

Cette effusion naturelle du sentiment religieux est absolument contraire au secret de l'intériorité kierkegaardienne; la relation religieuse ne peut s'exprimer directement, c'est-à-dire objectivement. Il y a communication directe chez l'un, communication par essence indirecte chez l'autre (s'il y a communication). Car Kierkegaard a bien conscience lui aussi des dangers de l'hermétisme auquel pourrait aboutir le repli sur soi de l'intériorité religieuse (le démoniaque), mais les principes mêmes de la relation religieuse interdisent une expression effusive et directe. Le croyant singulier ne peut non plus chercher chez les autres une confirmation, ce qui relativiserait la radicalité de sa décision de croire; d'ailleurs, quand même le voudrait-il, il ne pourrait être satisfait, puisqu'il se heurterait évidemment à l'opacité de 
la conscience religieuse individuelle. Plus un individu s'enfonce dans la relation religieuse, plus il s'éloigne des autres ${ }^{10}$. La vérité les sépare, précisément parce que «la vérité est la subjectivité ». Il ne peut donc y avoir de communication directe de religieux à religieux. Pourtant, le croyant singulier a bien un rôle à jouer dans l'institution d'une religion authentique, au moins dans le «réveil de la foi ». Mais comment le peut-il si, par essence, sa relation à Dieu ne peut s'objectiver dans une expression directe? Cette objectivation, quand bien même elle deviendrait effective — ce qui est impossible —, ne serait de toute façon d'aucune utilité, puisque chacun doit établir pour soimême et par soi-même (c'est-à-dire à partir de soi) une telle relation.

Le lien communautaire qui doit constituer la religion positive doit donc en définitive être soumis à une interrogation profonde sur la nature de la communication religieuse. Il y a bien chez Kierkegaard l'idée critique selon laquelle les prêtres ne sont plus que des fonctionnaires et des traîtres (les termes sont, en fait, encore plus injurieux), qui ne suscitent plus rien chez les fidèles et même les détournent prudemment de la véritable relation religieuse, parce qu'ils ont peur de perdre des adeptes en leur montrant l'effrayante difficulté du christianisme. Mais cette critique ne s'accompagne pas de la promotion, si l'on peut dire, du "virtuose ", concept qui lui est d'autant plus étranger qu'il ne peut y voir que l'expression d'un dangereux illuminisme qu'il a violemment critiqué dans «l'affaire Adler" ${ }^{11}$ ». Si communauté il y a, elle ne peut se faire sur le fond d'une croyance partagée - ce qui exclut définitivement toute assimilation de la foi à un schème épistémique de croyance dans lequel le rapport à la vérité est attesté par son universel partage. En un mot, elle ne peut être une communauté de savoir ${ }^{12}$.

C'est même là le point essentiel: le défaut rédhibitoire de la religion instituée, c'est précisément de réduire la relation de foi à ce schème, pour pouvoir se transmettre. La priorité de la transmission directe implique en effet la primauté donnée à la doctrine (ensemble de propositions qui est

10. Voir Kierkegaard $(1977,229)$ : «L'intériorité de la vérité n'est pas cette camaraderie où deux intimes se promènent bras dessus, bras dessous, mais bien la séparation dans laquelle chacun existe pour soi dans le vrai."

11. Voir Kierkegaard (1980). Le pasteur Adler prétendait avoir été l'objet d'une révélation par laquelle Dieu lui avait, entre autres, intimé l'ordre de détruire ses ouvrages de Hegel. Il fut finalement sanctionné par l'Église danoise. Kierkegaard se passionna pour le cas, au point d'écrire un long ouvrage (qu'il ne publia pas) dans lequel il prenait clairement position contre l'illuminisme ridicule, contradictoire et dangereux, du pasteur.

12. La religiosité de Schleiermacher s'oppose elle aussi à cette communauté de savoir qui est au contraire revendiquée par sa Dialektik. 
l'objet d'une relation de savoir) sur le Maître (qui implique par essence une relation de singulier à singulier, radicalement différente d'un schème épistémique): toute communauté se forme sur le fond d'un savoir en commun. Mais précisément la foi ne relève pas de ce rapport de type gnoséologique; et elle ne rapproche pas les individus dans un savoir commun, mais les éloigne dans une relation intensive et singularisante. Cependant, même débarrassée de ce schème, la relation pourrait tout de même impliquer une communication directe, comme le montre l'exemple de Schleiermacher pour qui la religion ne relève ni de la connaissance métaphysique ni de l'éthique. Or, à l'inverse de l'effusion (mouvement vers l'extérieur), la religiosité kierkegaardienne est un approfondissement de l'intériorité (mouvement vers l'intérieur).

Le long travail critique de Kierkegaard sur la notion de «témoin de la vérité ", qui l'entraînera d'ailleurs dans une polémique directe avec l'Église, lui interdit d'offrir cette qualité à la «simple» religiosité, fût-elle d'une intensité sans précédent. L'esprit religieux n'est pas apôtre pour autant. Il n'empêche que le témoignage peut bien constituer a minima un lien de reconnaissance, sans que pour autant il ait un effet de transmission directe de la foi ou même de mise en commun de l'expérience. Certes, ce témoignage peut prendre des marques visibles, dont la persécution et le martyre sont les plus flagrantes - mais ceci laisse dans l'ombre l'essentiel : la nature même de la relation intérieure. Sous quelle forme s'attestera donc cette relation, attestation qui pourrait ainsi établir quelque chose comme une communauté paradoxale de religieux isolés?

\section{La communauté discursive}

La solution pourrait se trouver dans le mode même d'expression de ce témoignage, lequel, chez Kierkegaard, doit être indexé sur le principe d'une communication nécessairement indirecte. L'effectivité de la religion, une fois remises en question l'institution et la communauté objectives qui prétendaient l'assumer, repose sur la forme d'expression : ce qui se substitue au cadre épistémique de la croyance partagée, c'est un espace de relations bilatérales fondées sur un style d'expression. En un mot: pas de communauté logique, universelle et objective, mais un réseau de relations ponctuelles, réitérées et singularisées, tissé par des règles discursives. C'est le discours qui établit la communauté (religieuse), conçu comme une communication dont les règles de construction et de signification ne relèvent plus de la logique de la connaissance, mais de la pragmatique. Un discours qui relève d'un art. 
Aussi bien ne sera-t-on pas étonné de voir Schleiermacher déclarer: "Voilà pourquoi il est impossible d'exprimer et de communiquer la religion autrement que sous la forme de l'art oratoire, avec toute la discipline et tout l'art du langage, et en se plaisant à mettre à contribution tous les arts susceptibles de seconder le discours dans ce qu'il a de fugace et de mouvant » (Schleiermacher 2004, 103). On ne s'étonnera pas, non seulement de le voir mobiliser les arts rhétoriques de la parole (et même le poétique), mais aussi insister spécialement sur l'art oratoire, c'est-à-dire une communication qui met en présence les interlocuteurs, qui prend en charge le caractère vivant et communicatif, sensible (phonique), de l'expression du sentiment religieux. Dès l'ouverture des Discours, il assurait le caractère rhétorique de la communication religieuse (27).

Mais on ne s'étonnera pas non plus de voir Kierkegaard, qui doit lui aussi penser le lien à partir de l'art du discours, absolument hostile à cet art oratoire (bien qu'il insiste sur la communication orale), au style fleuri, emphatique, sublime, artiste, des pasteurs, tout en établissant les principes d'un autre art de la communication religieuse. Il ne propose pas ce contremodèle en raison simplement du contenu du discours religieux, avec cette idée que la tâche n'est pas de montrer le sublime de la religion mais au contraire son caractère effrayant et difficile, mais aussi, et peut-être d'abord, pour des raisons formelles de communication. Cet art de la parole est quelque chose comme une rhétorique anti-rhétorique, puisqu'il ne cherche pas à persuader et à gagner des adeptes, mais au contraire à repousser les auditeurs vers leur propre intériorité. Il est quelque chose, également, qui bloque l'effusion directe tout en portant à la représentation la relation singulière, pour autant qu'elle puisse être indiquée (et non pas dévoilée sans reste). Cet art trouve alors son achèvement dans l'écriture, et, pour tout dire, dans l'écriture kierkegaardienne elle-même.

À l'art oratoire de Schleiermacher, dont les discours de De la religion sont eux-mêmes des performances (puisqu'il s'agit d'une apologie), Kierkegaard oppose sa propre écriture, une écriture ultra-complexe fondée sur la communication indirecte à laquelle l'art du discours n'offre pas moins de recours. Pour tout dire, il faut peut-être voir dans l'écriture de soi kierkegaardienne la clef de cet art du discours : elle est bien «l'expression », nécessairement indirecte, de la relation religieuse singulière. Une écriture singulière, dans tous les sens du terme, qui ne peut être un témoignage direct de l'intériorité - et qui du coup réclame des instruments spécifiques d'exposition, la fiction par exemple et non, justement, une confession - 
mais qui n'en est pas moins expressive de la seule subjectivité individuelle. C'est, par cette écriture, la seule " communauté » religieuse qui se puisse fonder sur la singularité irréductible de la relation religieuse. Et c'est là le mode de manifestation paradoxale de cette relation. La littérature, comme le montre par exemple Crainte et tremblement, indique l'intériorité invisible du Chevalier de la foi.

La particularité formelle de cet art d'exposition indirecte du soi religieux établit alors une relation au lecteur elle-même inédite, lequel, par le moyen de cette exposition même, se voit contraint de s'intérioriser, contraint de se retourner vers lui-même et d'entamer pour lui-même le chemin du devenir soi - c'est la parole kierkegaardienne qui cette fois devient miroir. La littérature (ou plutôt l'usage kierkegaardien de la littérature) est une manière de transmission du secret qui le préserve, si le secret est bien ce qui ne se dit qu'à une seule personne à la fois. Et c'est dans le milieu créé par l'acte de lecture que s'établit cette communauté paradoxale d'individus isolés. D'une certaine manière, la religion de Kierkegaard est une communauté de lecteurs, de lecteurs isolés et même de plus en plus isolés — voilà pourquoi il n'est ni pasteur en chaire, ni professeur. Si l'Église de Schleiermacher ressemble à une salle de concert, celle de Kierkegaard obéirait plutôt au modèle de la bibliothèque.

L'idée d'une forme sectaire, au sens troeltschien, de la religion kierkegaardienne, que l'on pourrait évoquer en raison de l'accentuation du caractère intensif et qualitatif, est tentante mais elle est fausse, parce qu'elle suppose encore un lien communautaire direct. La communauté de lecteurs et d'écrivains qu'établissent les règles kierkegaardiennes de discours paraît d'un autre ordre - à distance, indirecte et isolante. Mais elle est bien réelle, puisqu'elle s'effectue dans l'acte d'écrire et dans celui de lire.

\section{Conclusion}

Ce dernier schéma, en tous les cas, invite à penser la religion comme forme de communalisation discursive - et non pas comme croyance partagée. Et c'est peut-être là l'enseignement commun de ces deux voies pourtant si divergentes, divergentes et même antipodiques, paradoxalement parce qu'elles partent de prémisses proches. Pour les deux penseurs que nous avons mis en regard, en effet, la conception d'un noyau nécessairement singulier de la religion - la relation singulière et singulatisante à l'Absolu - ne fait pas que rendre problématique l'idée même de religion comme phénomène social et même objectif. Dans les deux cas, cette conception 
trahit un abandon du schéma épistémique classique à partir duquel on pense généralement la communauté religieuse (la croyance partagée). La structure discursive qui s'y substitue alors - qui prend en compte à la fois le mode de relation à l'absolu et la nature de la relation possible entre individus religieux - révèle un autre schéma, à la fois herméneutique et pragmatique, Schleiermacher et Kierkegaard fournissant, ici encore, deux versions possibles et fécondes de l'herméneutique philosophique. Cette substitution d'un schéma à un autre modifie ainsi profondément la conception de l'espace religieux. Un espace discursif, alors, mais bien paradoxal, car il n'est pas même organisé par un discours commun, mais comme réseau de relations établi par des performances de discours et des actes de lecture.

\section{Références}

Clair, A. (1993), Penser le singulier, Paris, Cerf.

Delecroix, V (2002), "Quelques traits d'une herméneutique kierkegaardienne ", Revue des Sciences philosophiques et théologiques, 86/2, p. 243-256.

KierkegaARd, S. (1972) [danois 1843], Crainte et tremblement, trad. P.-H. Tisseau et E.-M. Jacquet-Tisseau, dans Euvres complètes, t. v, Paris, Éditions de l'Orante.

Kierkegaard, S. (1977) [danois 1846], Post-Scriptum aux Miettes philosophiques, trad. P.-H. Tisseau et E.-M. Jacquet-Tisseau, in CEuvres complètes, t. x, Paris, Éditions de l'Orante.

KierkegaARd, S. (1980) [danois 1846-1847], Le livre sur Adler, trad. P.-H. Tisseau et E.-M. Jacquet-Tisseau, in Euvres complètes, t. XII, Paris, Éditions de l'Orante.

KiERKegaARD, S. (2006) [danois 1850], Exercice en christianisme, trad. v. Delecroix, Paris, Éditions du félin (Les marches du temps).

SCHLEIERMACHER, F. (2004) [allemand 1799], De la religion. Discours aux personnes cultivées d'entre ses mépriseurs, trad. B. Reymond, Paris, Van Dieren (Références théologiques).

Simon, M. (1974), La philosophie de la religion dans l'œuvre de Schleiermacher, Paris, Vrin. 


\section{Résumé}

À trop faire porter l'accent sur la relation individuelle et subjective à son objet, la pensée philosophique qui s'attache au religieux peut en venir à des cas limites où la nature sociale de la religion lui devient, d'un point de vue épistémologique, difficilement visible et pensable. On se proposera ici d'examiner les formes de cette articulation problématique entre expérience singulière et communautés objectives dans le cadre d'une comparaison entre deux de ces cas limites: Schleiermacher et Kierkegaard.

\section{Abstract}

By putting too much emphasis on the individual and subjective relationship to its object, the philosophy of religion can reach borderline instances where, from an epistemological point of view, the social nature of religion becomes hardly visible and understandable. We propose to examine the problematic manner in which individual experience and objectives communities are articulated by comparing two such borderline instances: Schleiermacher and Kierkegaard. 